\title{
Adaptive Filter Based PN Code Phase Acquisition Under Frequency Selective Rayleigh Fading Channels
}

\author{
Donghoon Lee*, Jeongchang Kim, Kyungwhoon Cheun
}

\begin{abstract}
A hybrid PN code phase acquisition system based on a least-mean-square adaptive filter, interpreted as a channel estimator is proposed and analyzed for direct-sequence spread-spectrum systems under frequency selective Rayleigh fading channels. Closed form expressions are derived for the filter tap weights and detection/false alarm probabilities. Compared to previously proposed systems, the proposed system achieves smaller mean acquisition times, is more robust to the operating signal-to-noise ratio and allows for multiplication free tap weight updates.
\end{abstract}

Key Words : Acquisition, adaptive filters, direct-sequence spread-spectrum, multipath channels

\section{I . Introduction}

There have been numerous attempts to improve the PN code phase acquisition performance of direct-sequence spread-spectrum (DSSS) receivers under frequency selective multipath fading channels. In [1], an optimal detection rule based on the maximum-likelihood principle was derived for parallel acquisition systems. In [2], chip level post-detection integration method was suggested in order to improve the performance of serial matched filter acquisition systems under multipath channels. On a different note, a partially parallel, hybrid acquisition system based on a least-mean-square (LMS) adaptive equalizer was proposed in [3-5]. Although specific PN code phase search strategies proposed in [1-5] are different, a commonality is that some form of windowing is employed in order to collect the signal power spread over the multipath components and seek diversity.

In this paper, we propose a novel hybrid PN code phase acquisition system based on an LMS adaptive filter structure interpreted as a channel estimator and analyze its performance. In contrast to the equalizer based structure of [3-5], the proposed structure takes the locally generated PN code as input (observation) to the adaptive filter and the received signal as the reference (training) signal (Fig. 1). Owing to this role reversal, the proposed system achieves smaller mean acquisition times and is more robust to the operating signal-to-noise ratio (SNR). Also, the new architecture allows for multiplication free tap weight updates and only one received sample per chip is required which significantly reduces implementation complexity.

The remainder of the paper is organized as follows. In Section II, the assumed channel

※ This research was supported by the MSIP(Ministry of Science, ICT\&Future Planning), Korea, under the ITRC(Information Technology Research Center) support program (NIPA-2013- H0301-13-1001) supervised by the NIPA(National IT Industry Promotion Agency)

※ This paper was presented in part at the 2006 International Symposium on Information Theory and its Applications, COEX, Seoul, Republic of Korea, Oct. 29-Nov. 1, 2006.

- First Author : Samsung Electronics, donghoon72.lee@samsung.com, Regular Member

- Corresponding Author : Division of Information Technology Convergence Engineering, Pohang University of Science and Technology (POSTECH), cheun@postech.ac.kr, Lifelong Member

* Department of Electronics and Communications Engineering, Korea Maritime University, jchkim@hhu.ac.kr, Lifelong Member 논문번호 : KICS2013-02-089, 접수일자 : 2013년 2월 12일, 최종논문접수일자 : 2013년 4월 17일 


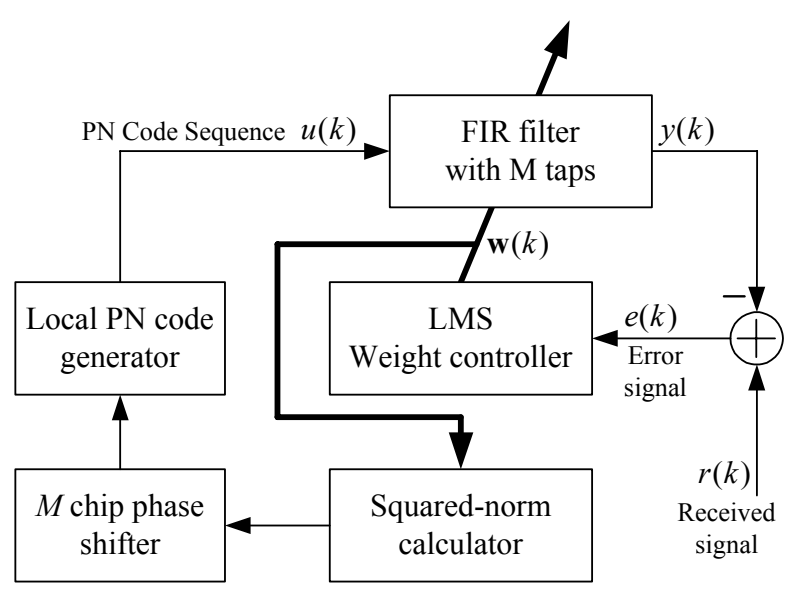

Fig. 1. Proposed PN code acquisition system.

model and the proposed system architecture are described. It is also shown that the Wiener-Hopf solution $^{[6]}$ to the tap weights for the proposed architecture is equal to the channel response covered by the search window. In Section III, the transient response for the tap weights are derived. Closed form expressions are derived for the tap weights after $N_{d}$ iterations which are modeled as independent zero mean complex Gaussian RVs. The Gaussian model for the tap weights is then used to derive closed form expressions for the false alarm and detection probabilities and the mean acquisition time. In Section IV, computer simulation results are provided to verify the accuracy of the derived analytical results. The results indicate that the derived analytical results for the detection and false alarm probabilities are indeed very accurate and that the proposed algorithm gives significantly improved performance compared to the equalizer based architecture. The results also verify that the proposed system is quite insensitive to the SNR. Finally, conclusions are drawn in Section $\mathrm{V}$.

\section{Proposed PN Code Phase Acquisition System}

The architecture of the proposed PN code phase acquisition system is depicted in Fig. 1 which is based on an LMS type adaptive filter updating the tap weight vector according to the stochastic gradient algorithm ${ }^{[6]}$. The adaptive filter attempts to minimize the mean-squared-error (MSE) between the filter output and the reference signal. Note that roles played by the locally generated PN code and the received signal are reversed compared to the equalizer based acquisition system proposed in [3-5]. Let $\mathrm{u}(k)=[u(k) u(k-1) \cdots u(k-M+1)]^{T} \quad$ be the contents of the adaptive filter of length $M$ at time $k$ where $u(i)$ is the locally generated PN sequence modeled as a complex i.i.d. (independent and identically distributed) random sequence with real and imaginary parts taking on the values of $\pm 1 / \sqrt{2}$ with equal probability. Then, the filter output at time $k$ is computed as

$$
y(k)=\mathrm{w}(k)^{H} \mathrm{u}(k)
$$

where $\mathbf{w}(k)=\left[w_{0}(k) w_{1}(k) \cdots w_{M-1}(k)\right]^{T}$ denotes the filter tap weight vector at time $k$. Here and in the following, $\mathrm{a}^{T}$ and $\mathrm{a}^{H}$ denote the transpose and the Hermitian transpose of the complex vector $\mathrm{a}$, respectively. The reference signal for the adaptive filter is the complex baseband received signal $r(t)$ sampled at the chip rate of $1 / T_{c}$, given as

$$
r(k)=r\left(k T_{c}\right)=\sum_{m=-\infty}^{\infty} h(m) u(k+D-m)+N(k) .
$$

Here, $N(k)=N_{I}(k)+j N_{Q}(k)$ are i.i.d. complex zero mean Gaussian RVs due to additive white Gaussian noise (AWGN) where $N_{I}(k)$ and $N_{Q}(k)$ are i.i.d. zero mean Gaussian RVs with variance $N_{0} / 2$. Also, $D$ denotes the code phase difference, in number of chips, between the local PN code generator and the received signal. The channel is modeled as having the following baseband equivalent impulse response

$$
h(m)=\sum_{l=0}^{L_{p}-1} \alpha_{l} e^{j \theta_{l}} p\left(m T_{c}-\tau_{l}\right)
$$

where $p(t)$ represents a raised-cosine pulse with roll-off factor $\beta, \alpha_{l}$ are independent Rayleigh 
RVs with $\sum_{l=0}^{L_{p}-1} E\left[\alpha_{l}^{2}\right]=E_{c}$, the average total received energy per chip and $\theta_{l}$ are i.i.d. RVs uniformly distributed on $\left[\begin{array}{ll}0 & 2 \pi\end{array}\right)$. Also, $\tau_{l}$ are the delays of the $L_{p}$ separable multipath components with $0 \leq \tau_{0}<\tau_{1}<\cdots<\tau_{L_{p}-1}$ and $\tau_{i+1}-\tau_{i} \geq T_{c}$, $i=0,1, \cdots, L_{p}-2$. We assume that $\alpha_{l}$ and $\theta_{l}$ are approximately constant within a search dwell time but are independent among dwell times ${ }^{[7]}$.

Since the PN code is assumed to be i.i.d. with unit variance, the correlation matrix of the input vector, $\mathrm{R}=E\left\{\mathrm{u}(k) \mathrm{u}(k)^{H}\right\}$, is an $M \times M$ identity matrix, $I_{\mathrm{M}}$. Thus, the optimum value of the tap weight vector, $\mathrm{w}_{\mathrm{opt}}$ that minimizes the steady-state MSE is given by the Wiener-Hopf solution ${ }^{[6]}$ as

$\mathrm{w}_{\mathrm{opt}}=\mathrm{R}^{-1} \mathrm{P}=\mathrm{P}$

where $\mathrm{P}=E\left\{\mathrm{u}(\mathrm{k}) \mathrm{r}^{*}(\mathrm{k})\right\} \quad$ is the $\quad M \times 1$ cross-correlation vector which can easily be shown to be equal to $\left[h^{*}(D) h^{*}(D+1) \cdots h^{*}(D+M-1)\right]^{T}$. Hence, the optimum tap weight vector $\mathrm{w}_{\mathrm{opt}}$ is the complex conjugate of a portion of the channel response $h(k)$ covered by the current search window. This leads to the interpretation of the proposed adaptive filter as a channel estimator.

Note that since the optimum tap weights are obtained from the cross-correlation between the received signals and the reference $\mathrm{PN}$ code, the proposed system can be viewed as the conventional PN code phase acquisition system based on the cross-correlation. In the proposed system, however, the tap weights are obtained from the standard LMS tap weight update equation of the role reversed adaptive filter as shown in Fig. 1. Using the described adaptive filter, the proposed system performs a hybrid serial/parallel search of the received $\mathrm{PN}$ code phase similar to that of [3]. The candidate PN code phase to be searched are divided into $q$ disjoint cells each covering $M$ code phase hypotheses and the cells are tested sequentially. For each test, the squared norm of the tap weight vector is calculated at the end of dwell time and compared to a decision threshold.

If the squared norm is larger than the threshold, the cell under test is assumed to be the in-phase cell $\left(\mathrm{H}_{1}\right.$ hypothesis). Otherwise, the cell is assumed to be an out-of-phase cell $\left(\mathrm{H}_{0}\right.$ hypothesis) and the phase of the locally generated PN code is shifted by $M$ chips to test the next candidate cell to be tested. This process proceeds until an $\mathrm{H}_{1}$ hypothesis is declared or a time-out condition is met.

\section{Performance Analysis}

In this section, we derive closed form expressions for the transient response of the tap weights and the detection and false alarm probabilities of the proposed PN code phase acquisition scheme under frequency selective Rayleigh fading channels. In order to make the analysis tractable, the following two assumptions are made. First, the delays of the multipath components, $\tau_{l}$ are assumed to be integer multiples of the chip duration, $T_{c}$ and coincides with the sampling phase of the sampler, i.e., the received signal is sampled at the eye open phases. This assumption leads to an optimistic performance prediction. However, we show in the numerical results section that the SNR loss suffered by the proposed acquisition algorithm when all of the multipath components have a half chip timing offset from the assumed sampling phase (the worst case), is negligible. The second assumption is that the search window size of the adaptive filter is larger than the channel delay spread, i.e., $M T_{c}>\tau_{L_{p}-1}-\tau_{0}$ and that all multipath components are located in a unique search window. That is, there is only one in-phase cell among the $q$ cells to be searched. This assumption results in higher mean acquisition times compared to the case when the multipath components are spread over multiple consecutive cells. The difference, however, is usually small enough in practical situations that considering only 
the former case leads to a simple but a plausible estimate of the mean acquisition time $^{[3]}$. For convenience of presentation, the setup is that $D=0$ corresponds to the $\mathrm{H}_{1}$ hypothesis and $D=i M, i=1,2, \cdots, q-1$ are the $\mathrm{H}_{0}$ hypotheses.

\subsection{Transient response}

The standard LMS tap weight update equation is given by ${ }^{[6]}$

$$
\begin{aligned}
\mathrm{w}(k+1) & =\mathrm{w}(k)+\mu \mathrm{u}(k) e^{*}(k) \\
= & \mathrm{w}(k)-\mu \mathrm{u}(k) y^{*}(k)+\mu \mathrm{u}(k) r^{*}(k) \\
= & \mathrm{w}(k)-\mu \mathrm{u}(k) \mathrm{u}^{H}(k) \mathrm{w}(k)+\mu \mathrm{u}(k) r^{*}(k) \\
= & {\left[\mathrm{I}_{M}-\mu \mathrm{u}(k) \mathbf{u}^{H}(k)\right] \mathrm{w}(k)+\mu \mathrm{u}(k) r^{*}(k) }
\end{aligned}
$$

where $0<\mu<1$ is the adaptation step size and $e(k)=(r(k)-y(k))$ is the error signal generated by the adaptive filter. Note that $\mathrm{u}(k)$ represents the complex binary, locally generated PN sequence as assumed in Section II. Hence, constraining $\mu$ to be a negative power of 2 allows for a multiplication free coefficient update implementation using only bit shift and addition operations.

The step size in (5) is usually set small enough to average out the gradient noise, particularly when the received SNR (before despreading) is very small, as is typical in DSSS receivers. In such a case, the system matrix $\left[\mathrm{I}_{M}-\mu \mathrm{u}(k) \mathrm{u}^{H}(k)\right]$ in (5) can be safely replaced by its ensemble average given by $\left[\mathrm{I}_{M}-\mu \mathrm{R}\right]^{[6]}$. Since $R=I_{M}$ in the proposed system, the vector tap weight update equation (5) can be decoupled as follows:

$$
\begin{array}{r}
w_{i}(k+1)=(1-\mu) w_{i}(k)+\mu u(k-i) r^{*}(k) \\
i=0,1, \cdots, M-1
\end{array}
$$

Hence, the individual tap weights can be viewed as being obtained by passing the output of the corresponding PN correlator through a first-order low pass filter with transfer function

$$
H(z)=\frac{\mu}{z-(1-\mu)}
$$

Once acquisition is obtained, this allows the proposed system to continuously estimate and track the channel variations with an appropriately chosen $\mu$. This suggests that in a continuous operational mode, the proposed system may be utilized to perform continuous code phase tracking in addition to one shot code phase acquisition ${ }^{[8]}$. Also, note that the steady-state tap weight vector approximates, and thus, provides an estimate of $\mathrm{w}_{\mathrm{opt}}$. Since $\mathrm{w}_{\mathrm{opt}}=\left[h^{*}(D) h^{*}(D+1) \cdots h^{*}(D+M-1)\right]^{T}$ represents the channel response covered by the current search/tracking window, the steady-state tap weight vector may also be used as the combining weights for a RAKE receiver, another interesting advantage obtainable with the proposed system $^{[8,12]}$.

For PN code phase acquisition, the proposed system performs the tap weight updates following (6) for a given fixed adaptation period (dwell time) for each cell to be tested. Assuming that the tap weight update starts at time 0 and the initial tap weight vector is the all zeros vector, the $i$ th tap weight, $i=0,1, \cdots, M-1$ after $N_{d}$ iterations may be expressed as follows:

$$
w_{i}\left(N_{d}\right)=\mu \sum_{n=0}^{N_{d}-1}(1-\mu)^{N_{d}-n-1} u(n-i) r^{*}(n) .
$$

Then, we can denote the tap weight vector after $N_{d}$ iterations as $\mathrm{w}\left(N_{d}\right)=\left[w_{0}\left(N_{d}\right) \cdots w_{M-1}\left(N_{d}\right)\right]$. Via straightforward computations, (8) may further be simplified as

$$
w_{i}\left(N_{d}\right)=\left[1-(1-\mu)^{N_{d}}\right] h^{*}(i+D)+Z_{i}
$$

where

$$
\begin{aligned}
Z_{i}=\mu \sum_{n=0}^{N_{d}-1}(1-\mu)^{N_{d}-n-1} u(n-i) & {\left[N^{*}(n)+\sum_{\substack{m=-\infty \\
m \neq i+D}}^{\infty} h^{*}(m) u^{*}(n+D-m)\right] }
\end{aligned}
$$

Note that $Z_{i}$ represents the contribution of the AWGN and multipath interference, modeled as an 
independent zero mean complex Gaussian RV with variance.

$$
\begin{aligned}
\sigma_{Z_{i}}^{2} & =E\left[\left|Z_{i}\right|^{2}\right] \\
& =\frac{\mu}{2-\mu}\left[1-(1-\mu)^{2 N_{d}}\right]\left(P_{R}-\sigma_{h, i}^{2}\right)
\end{aligned}
$$

We make the usual assumption ${ }^{[9-13]}$ that the real and imaginary parts of $Z_{i}$ are uncorrelated with an identical variance of $\frac{1}{2} \sigma_{Z_{i}}^{2}$ and $P_{R}=E_{c}+N_{0}$ is the total received power in $r(k)$ and $\sigma_{h, i}^{2}=E\left[|h(i+D)|^{2}\right] . \quad$ For a given channel response within a search dwell time, averaging both sides of (9),

$E\left\{w_{i}\left(N_{d}\right)\right\}=\left[1-(1-\mu)^{N_{d}}\right] h^{*}(i+D)$.

Note that $\lim _{N_{d} \rightarrow \infty} E\left\{w_{i}\left(N_{d}\right)\right\}=h^{*}(i+D)$ which is the solution to the Wiener-Hopf equation found in Section II. We also observe that (12) does not depend on the SNR which indicates that, at least in terms of the progression of the average tap weights, the system is insensitive to the operational SNR.

At the end of each dwell time, the squared norm of the tap weight vector is computed and compared to a threshold. Given the decision variable $\quad \Gamma=\sum_{i=0}^{M-1}\left|w_{i}\left(N_{d}\right)\right|^{2} \quad$ and $\quad$ a detection threshold $\eta$, a false alarm occurs when $\Gamma>\eta$ under the $\mathrm{H}_{0}$ hypothesis with probability $P_{F A}=\operatorname{Pr}\left\{\Gamma>\eta \mid \mathrm{H}_{0}\right\}$ and detection occurs when $\Gamma>\eta \quad$ under the $\mathrm{H}_{1}$ hypothesis with $P_{D}=\operatorname{Pr}\left\{\Gamma>\eta \mid \mathrm{H}_{1}\right\}^{[14]}$.

\subsection{False alarm probability}

Let $X_{i}, i=1,2, \cdots, N, N=$ even, be i.i.d. zero mean Gaussian RVs each with variance $\sigma^{2}$. Then, $Y=\sum_{i=1}^{N} X_{i}^{2} \quad$ follows the central chi-squared distribution with $N$ degrees of freedom with the probability density function and the cumulative distribution function given by

$f\left(y, L, \sigma^{2}\right)=\frac{1}{\sigma^{2 L} 2^{L}(L-1) !} y^{L-1} e^{-y / 2 \sigma^{2}}$

and

$$
F\left(y, L, \sigma^{2}\right)=1-e^{-y / 2 \sigma^{2}} \sum_{k=0}^{L-1} \frac{1}{k !}\left(\frac{y}{2 \sigma^{2}}\right)^{k}
$$

where $L=N / 2$.

Recall that under the $\mathrm{H}_{0}$ hypothesis, $w_{i}\left(N_{d}\right)=Z_{i}$ with the following variance per dimension

$\sigma_{\mathrm{H}_{0}}^{2}=\frac{1}{2}\left(\frac{\mu}{2-\mu}\right)\left[\left(1-(1-\mu)^{2 N_{d}}\right] P_{R}\right.$

Then, the decision variable $\Gamma$ is a sum of $M$, i.i.d. central chi-squared RVs with 2 degrees of freedom which in turn follows the central chi-squared distribution with $2 M$ degrees of freedom with $\sigma^{2}=\sigma_{\mathrm{H}_{0}}^{2}$. Strictly speaking, $w_{i}$ in (9) are not mutually independent due to the interpath interference ${ }^{[12]}$, but usually, the effect of the interpath interference is small enough that it can safely be neglected. As a result, the false alarm probability is given as ${ }^{[13]}$

$$
P_{F A}=1-F\left(\eta, M, \sigma_{\mathrm{H}_{0}}^{2}\right) .
$$

\subsection{Detection probability}

Under the $\mathrm{H}_{1}$ hypothesis, $w_{i}\left(N_{d}\right)=\left[1-(1-\mu)^{N_{d}}\right] h^{*}(i)+Z_{i} \quad$ and $h(i)$, $i=0,1, \cdots, M-1$ are given by

$h(i)=\left\{\begin{array}{l}\alpha_{l} e^{j \theta_{l}}, l=0,1, \cdots, L_{p}-1, i=\tau_{l} / T_{c} \\ 0, \quad \text { otherwise }\end{array}\right.$

due to the assumption that $\tau_{l}$ are integer multiples of $T_{c}$ and coincides with the sampling phase of the sampler. The per dimension variance of $w_{i}\left(N_{d}\right)$ is then given by 
$\sigma_{w_{i}}^{2}=\left\{\begin{array}{c}\sigma_{\mathrm{H}_{1}, l}^{2}=\frac{1}{2}\left[1-(1-\mu)^{N_{d}}\right]^{2} E\left[\alpha_{l}^{2}\right]+\frac{1}{2} \sigma_{Z_{i}}^{2}, \\ l=0, \cdots, L_{p}-1, i=\tau_{l} / T_{c} \\ \sigma_{\mathrm{H}_{0}}^{2}, \quad \text { otherwise }\end{array}\right.$

The decision variable $\Gamma$ in this case is a sum of $\left(M-L_{p}\right)$, i.i.d. central chi-squared RVs with 2 degrees of freedom with $\sigma^{2}=\sigma_{\mathrm{H}_{0}}^{2}$, plus $L_{p}$ independent and non-identically distributed central chi-square RVs with 2 degrees of freedom with $\sigma^{2}=\sigma_{\mathrm{H}_{1}, l}^{2}, \quad l=0, \cdots, L_{p}-1$. As with the derivation of the false alarm probability, we assume that $w_{i}\left(N_{d}\right)$ are mutually independent. Then, the detection probability can be derived using the partial fraction expansion of the characteristic function of $\Gamma$ as described in [1]. The detection probability for the simple case of a uniform multipath intensity profile ( $E\left[\alpha_{0}^{2}\right]=E\left[\alpha_{1}^{2}\right]=\cdots=E\left[\alpha_{L_{p-1}}^{2}\right]=E_{c} / L_{p}$ and thus, $\sigma_{\mathrm{H}_{1}, l}^{2}=\sigma_{\mathrm{H}_{1}}^{2}=\frac{1}{2}\left[1-(1-\mu)^{N_{d}}\right]^{2} \frac{E_{c}}{L_{p}}$ $\left.+\frac{1}{2} \frac{\mu}{2-\mu}\left[1-(1-\mu)^{2 N_{d}}\right]\left(P_{R}-\frac{E_{c}}{L_{p}}\right),{ }^{\forall} l\right)$ may be derived as follows ${ }^{[1]}$ :

$P_{D}=1-\sum_{l=1}^{M-L_{p}} a_{l} F\left(\eta, l, \sigma_{\mathrm{H}_{0}}^{2}\right)-\sum_{l=1}^{L_{p}} b_{l} F\left(\eta, l, \sigma_{\mathrm{H}_{1}}^{2}\right)$

where $a_{l}$ and $b_{l}$ are given by

$$
\begin{gathered}
a_{l}=\left(\begin{array}{c}
M-l-1 \\
L_{p}-1
\end{array}\right)(-1)^{M-L_{p}-l} \frac{\left(\sigma_{\mathrm{H}_{1}}^{2} / \sigma_{\mathrm{H}_{0}}^{2}\right)^{M-L_{p}-l}}{\left(1-\sigma_{\mathrm{H}_{1}}^{2} / \sigma_{\mathrm{H}_{0}}^{2}\right)^{M-l}}, \\
l=1,2, \cdots, M-L_{p}, \\
b_{l}=\left(\begin{array}{c}
M-l-1 \\
M-L_{p}-1
\end{array}\right)(-1)^{L_{p}-l} \frac{\left(\sigma_{\mathrm{H}_{0}}^{2} / \sigma_{\mathrm{H}_{1}}^{2}\right)^{L_{p}-l}}{\left(1-\sigma_{\mathrm{H}_{0}}^{2} / \sigma_{\mathrm{H}_{1}}^{2}\right)^{M-l}}, \\
l=1,2, \cdots, L_{p} .
\end{gathered}
$$

Note that the uniform multipath intensity profile corresponds to the worst case condition from the acquisition point of view when $L_{p} \gg 1^{[2]}$. We refer the readers to [1] for the detection probabilities corresponding to various multipath intensity profiles.

\subsection{Mean acquisition time}

In order to simplify the derivation, we assume that when an $\mathrm{H}_{1}$ hypothesis is declared, the proposed system directly turns control over to a code tracking loop without verification ${ }^{[15]}$. Also, we assume no additional in-depth search stage selecting useful code phases among the $M$ possible candidates before assigning them to the code tracking loops. Then, the mean acquisition time can be found via the standard Markov chain approach $^{[15]}$ for a single dwell, straight line search system with a uniform a priori distribution to be

$\overline{T_{a c q}}=\frac{2+\left(2-P_{D}\right)(q-1)\left(1+K_{p} P_{F A}\right)}{2 P_{D}} N_{d} T_{c}$

where $K_{p}$ is the false alarm penalty factor.

\section{Numerical results}

For all numerical results presented in this section, we assume $M=11$, a raised-cosine overall chip pulse shape with a roll-off factor of $\beta=0.2$ and a uniform multipath intensity profile. The channel is assumed to be a slow fading in the sense that the channel response does not vary significantly during an acquisition process. As previously mentioned, all multipath components are assumed to be located within a single search cell (the $\mathrm{H}_{1}$ hypothesis) and are separated by integer multiples of $T_{c}$. It is also assumed that the carrier frequency offset is small enough so that the carrier phase rotation within a given dwell time is constant.

Fig. 2 shows analytic and simulated receiver operating characteristics (ROCs) curves for the proposed system for $L_{p}=2$ with a uniform multipath intensity profile and various values of $E_{c} / N_{0}$ and $N_{d}$. The delays of the multipath 


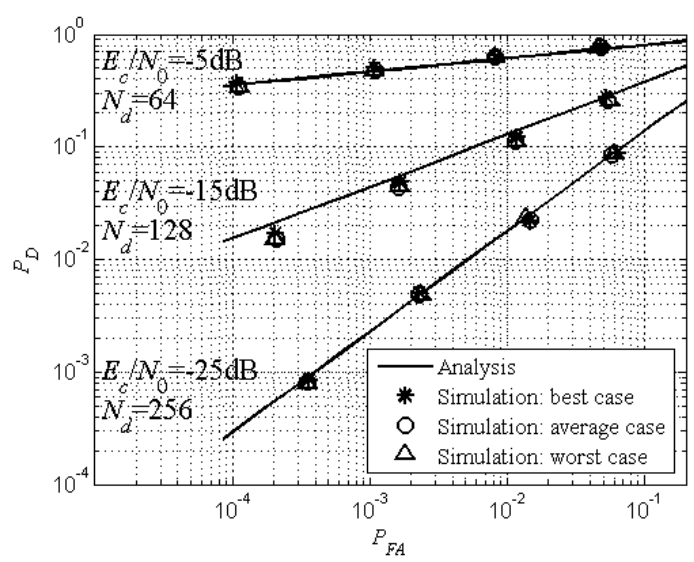

Fig. 2. Detection versus the false alarm probabilities for the proposed system for $L_{p}=2$ with a uniform multipath intensity profile, $M=11$ and $\mu=2^{-7}$.

components are 5 and 7 chip durations for the first and second paths, respectively. We designate the case when all the multipath components have a zero sampling phase offset as the best case, and the case when all the multipath components have a sampling phase offset of a half a chip duration as the worst case. The case when the sampling phase offsets of the multipath components are uniformly distributed on $\left(-T_{c} / 2 T_{c} / 2\right]$, is referred to as the average case. Clearly, the effect of the sampling phase offset is negligible, and hence, the sampling rate of one sample per chip is sufficient. This is mainly due to the fact that the proposed system performs a window based search and thus the energy of a chip pulse within the search window is insensitive to the sampling phase if the size of the search window is sufficiently larger than the main lobe of the chip pulse shape. Requiring no more than one sample per chip duration is advantageous in terms of the required sampling rate and the number of required tap coefficients to realize a given search window size. As described in Section III, no multiplication operations are required for tap weight updates of the proposed scheme. Tap weight updates can be implemented using only bit shift and addition operations. During dwell time, the required number of addition operations is $M N_{d}$. Conventional serial search systems, on the other hand, usually require

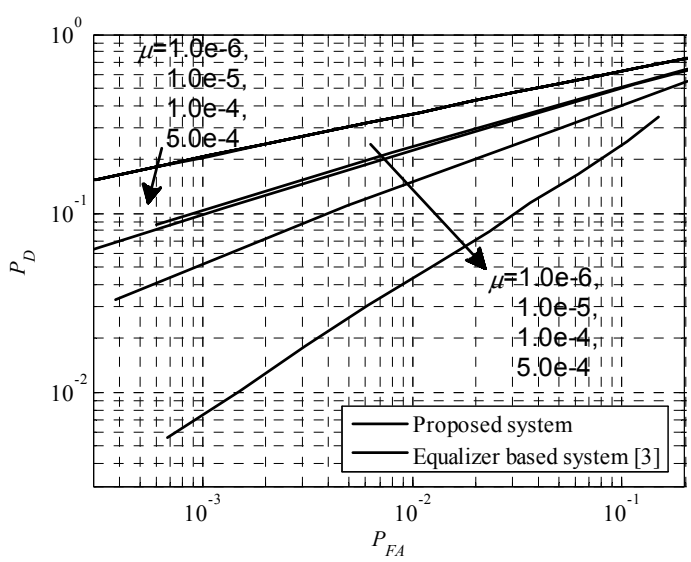

Fig. 3. Detection versus the false alarm probabilities for the proposed system and the equalizer based system of [3] for $L_{p}=2$ with a given uniform multipath intensity profile, $M=11$ and $E_{c} / N_{0}=-15 \mathrm{~dB}, N_{d}=256$.

higher sampling rates (and thus larger number of hypothesis cells to be tested) in order to avoid serious performance losses ${ }^{[7]}$, resulting in higher computational load and larger mean acquisition times. Finally, we observe that the analytical results derived in (16) and (19) very accurately predict the simulation results.

Fig. 3 shows the ROCs curves for the proposed system and the equalizer based system of [3] as a function of $\mu$ for $E_{c} / N_{0}=-15 \mathrm{~dB}$ and $N_{d}=256$. The uniform two ray channel model used in Fig. 2 is adopted. We observe that the ROCs of the proposed system are nearly invariable over a more wide range of $\mu$ compared to the equalizer based system of [3]. Also, the equalizer based system of [3] has worse ROCs than the proposed system. Hence, we can expect that the proposed system achieves smaller mean acquisition times than the equalizer based system of [3] as shown in Fig. 4. Fig. 4 shows the minimum mean acquisition times (MMATs) obtainable with the proposed algorithm and the equalizer based system of [3] as a function of $\mu$ for $q=100, K_{p}=180$ and various values of $E_{c} / N_{0}$ and $N_{d}$.

The uniform two ray channel model used in Fig. 2 is adopted with the best sampling phase offset. We observe that compared to the equalizer based system, the proposed system shows nearly 


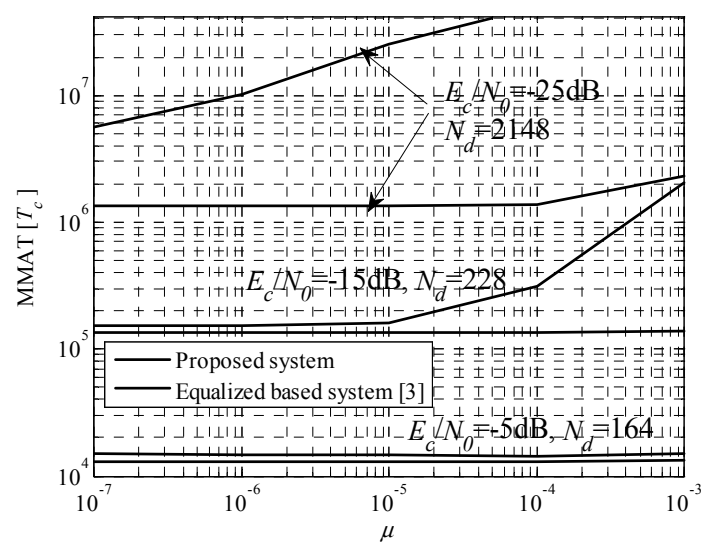

Fig. 4. Minimum mean acquisition time versus adaptation step size $\mu$ for $L_{p}=2$ with a given uniform multipath intensity profile, $M=11$ and $q=100, K_{p}=180$.

constant MMATs over a more wide range of $\mu$ for all cases considered and achieves smaller MMATs. This is due to the fact that for the equalizer based system of [3], the correlation matrix $\mathrm{R}$ determining the behavior of adaptive filters is a function of $E_{c} / N_{0}$. However, the proposed system has the correlation matrix $R=I$, and thus the behavior of adaptive filters of the proposed system is more robust to $E_{c} / N_{0}$. Also, it is clear that limiting $\mu$ to a power of 2 will cause no noticeable degradation in performance, which allows for a multiplierless implementation of the tap coefficient updates.

\section{Conclusions}

We proposed a hybrid PN code phase acquisition system based on an LMS adaptive channel estimator architecture for DSSS receivers under frequency selective Rayleigh fading channels. Also, the tap weight of the proposed system was obtained from the standard LMS tap weight update equation. The average tap weight is clearly independent of the received SNR and are shown to converge to the channel response covered by the current search window as the adaptation progresses. Hence, we may conclude that the proposed system should be much more robust to the specific operating conditions compared to the equalizer based system. Also, closed form expressions for the detection and false alarm probabilities were derived and their accuracies were assessed via simulations. Compared to the previously proposed adaptive channel equalizer based system, the proposed system achieves smaller mean acquisition times. Also, the fact that no multiplication operations are required for tap weight updates and only one received sample per chip is required results in a significantly decreased implementation complexity.

\section{References}

[1] R. R. Rick and L. B. Milstein, "Optimal decision strategies for acquisition of spread-spectrum signals in frequency-selective fading channels," IEEE Trans. Commun., vol. 46, no. 5, pp. 686-694, May 1998.

[2] J. Iinatti and M. Latva-aho, "A modified CLPDI for code acquisition in multipath channel," in Proc. IEEE PIMRC 2001, vol. 2, pp. 6-10, San Diego, U.S.A., Sep. 2001.

[3] M. G. El-Tarhuni and A. U. H. Sheikh, "Code acquisition of DS/SS signals in fading channels using an LMS adaptive filter," IEEE Commun. Lett., vol. 2, no. 4, pp. 85-88, April 1998.

[4] M. G. El-Tarhuni and A. U. H. Sheikh, "Application of adaptive filtering to direct-sequence spread-spectrum code acquisition," Wireless Personal Commun., vol. 8, no. 2, pp. 185-204, Sep. 1998.

[5] T. Yu, G. Park, D. Hong, and C. Kang, "Improved adaptive code acquisition scheme for practical DS/SS systems," in Proc. IEEE $60^{\text {th }}$ Veh. Tech. Conf., vol. 5, pp. 3743-3747, Los Angeles, U.S.A., Sep. 2004.

[6] S. Haykin, Adaptive Filter Theory, Englewood Cliffs, NJ: Prentice-Hall, 1986.

[7] A. J. Viterbi, CDMA Principles of Spread Spectrum Communications, Addison Wesley Publishing Company, 1995.

[8] D. Lee, "Symbol synchronization algorithms for OFDM and DS-SS systems under multipath fading channels," Ph.D. dissertion, 
Dept. Electron. Electrical Eng., Pohang University of Science and Technology (POSTECH), Pohang, Korea, 2002.

[9] M. B. Pursley, "Performance evaluation for phase-coded spread-spectrum multiple-access communication -- Part I: system analysis," IEEE Trans. Commun., vol. 25, no. 8, pp. 795-799, Aug. 1977.

[10] M. B. Pursley, D. V. Sarwate, and W. E. Stark, "Error probability for direct-sequence spread-spectrum multiple-access communications --Part I: upper and lower bounds," IEEE Trans. Commun., vol. 30, no. 5, pp. 975-984, May 1982.

[11] K. Cheun, Spread-Spectrum Communications: Introduction to Basic Concepts with Emphasis on Direct-Sequence Spreading, Pohang, Korea: POSTECH Press, 1995.

[12] K. Cheun, "Performance of direct-sequence spread-spectrum RAKE receivers with random spreading sequences," IEEE Trans. Commun., vol. 45, no. 9, pp. 1130-1143, Sep. 1997.

[13] J. Proakis, Digital Communications, New York: McGraw-Hill, 1989.

[14] K. H. Choi and K. W. Cheun, "PN code acquisition using instantaneous power-scaled detection threshold," in Proc. IEEK Conf., vol. 8, no. 1, pp. 162-166, Jan. 1998.

[15] J. K. Holmes, Coherent Spread Spectrum Systems, New York: John Wiely \& Songs, 1981.
Donghoon Lee

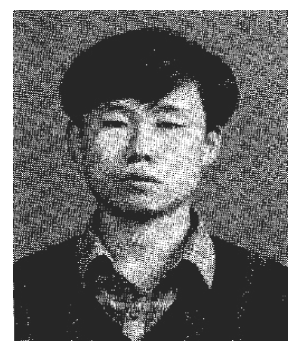

Donghoon Lee was born in Pusan, Korea, 1972 and received the B.S., M.S. and Ph.D. degrees in electronic and electrical engineering from Pohang University of Science and Technology (POSTECH), Pohang, in 1995, 1997, and 2003, respectively. In 2003, he joined Samsung Electronics Co., Ltd., Korea. His current research interests include synchronization for wireless modems, wireless LAN and VLSI design.

\section{Jeongchang Kim}

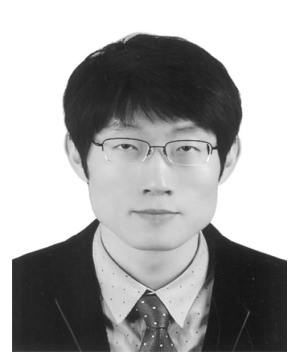

Jeongchang Kim was born in Daegu, Korea, on June 23, 1977. He received the B.S. degree in electronics, communication and radio engineering from Hanyang University, Seoul, Korea, in 2000, and M.S. and Ph.D. degrees from Pohang University of Science and Technology (POSTECH), Pohang, Korea, in 2002 and 2006, respectively, both in electronic and electrical engineering. From 2006 to 2008, he was a Full-Time Researcher at POSTECH, and from 2008 to 2009, he was a Research Assistant Professor. From 2009 and 2010, he was with the Broadcasting Systems Research Department at Electronics and Telecommunications Research Institute (ETRI) as a senior member of engineering staff. In 2010, he joined the Department of Electronics and Communications Engineering at Korea Maritime University, Busan, Korea, where he is currently an Associate Professor. His current research interests include MIMO, space-time codes, OFDM, digital communication systems. 


\section{Kyungwhoon Cheun}

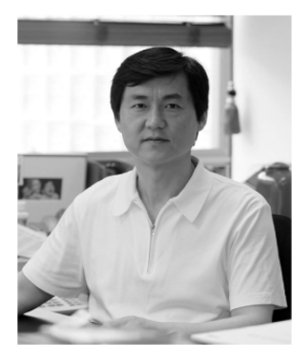

Kyungwhoon Cheun was

born in Seoul, Korea, on

December 16, 1962. He received his B.A. degree in electronics engineering from Seoul National University, in 1985, and M.S. and Ph.D. degrees from the University of Michigan, Ann Arbor, in 1987 and 1989, respectively, both in electrical engineering. From 1989 to 1991, he was with the Electrical Engineering Department, University of Delaware, Newark, as an Assistant Professor. In 1991, he joined the Division of Electrical and Computer Engineering, Pohang University of Science and Technology (POSTECH), Pohang, Korea, where he is currently a Professor and the Director of the Center for Broad-band OFDM Multiple Access (BrOMA) supported by the Ministry of Knowledge Economy (MKE), Korea. He has also served as an engineering consultant to various industries in the area of mobile communications and modem design and currently serves as the CTO at Pulsus Technologies, a fabless SOC company specializing in full digital amplifiers. His current research interests include OFDM, turbo and turbo-like codes, space-time codes, MIMO systems, software-defined radio and audio signal processing. 